\title{
Collimated, single-pass atom source from a pulsed alkali metal dispenser for laser-cooling experiments
}

\author{
Kevin L. Moore Thomas P. Purdy, Kater W. Murch, Sabrina Leslie, Subhadeep Gupta, and Dan M. Stamper-Kurn \\ University of California \\ 366 LeConte Hall, Berkeley, CA 94720
}

\begin{abstract}
We have developed an improved scheme for loading atoms into a magneto-optical trap (MOT) from a directed rubidium alkali metal dispenser in $<10^{-10}$ torr ultra-high vacuum conditions. A current-driven dispenser was surrounded with a cold absorbing "shroud" held at $\leq 0{ }^{\circ} \mathrm{C}$, pumping rubidium atoms not directed into the MOT. This nearly eliminates background atoms and reduces the detrimental rise in pressure normally associated with these devices. The system can be welldescribed as a current-controlled, rapidly-switched, two-temperature thermal beam, and was used to load a MOT with $3 \times 10^{8}$ atoms.
\end{abstract}

PACS numbers: $32.80 . P \mathrm{j}, 39.10 .+\mathrm{j}, 42.50 . \mathrm{Vk}$

\section{INTRODUCTION}

The first step in the construction of an atomic physics experiment is obtaining an appropriate source of atoms. Directed sources of atoms have a long and storied history [1]. A thermal beam of atoms is easily obtained from an oven or other gas source, though this inevitably involves a differential pumping scheme, a $1 / r^{2}$ decrease in atom flux with distance between the oven and the collection region, a mechanical shutter to quench the beam, and direct handling of a purified sample of the atom of interest. A Zeeman slower [2] can improve the flux of laser-cooled atoms from an oven, but suffers from the same drawbacks as an oven as well as the added complications of the magnetic design and the slowing beams. A multiply loaded magneto-optical trap (MOT) 3] initially loaded from a vapor cell is a widely-used source for cold atom experiments, but has the drawback of increased optical, electronic, and vacuum infrastructure. Light-induced atomic desorption (LIAD) [4], while not a collimated beam source, is an elegant technique which has recently been improved to yield very fast MOT loading rates [5]. However, the infrastructure necessary for fast LIAD is not always appropriate for other experimental requirements.

Alkali metal dispensers [6], or "getters," have emerged as a useful alternative to these sources [7, 8], requiring only a modest electric current $(<10 \mathrm{~A})$ for their operation. The driving current rapidly heats the dispenser causing a reduction reaction, inducing the $\mathrm{cm}$ scale devices to release an atomic vapor (rubidium, in our case) with a rapid turn-off time. The emitted atoms are quite hot, as the dispensers reach temperatures of $800{ }^{\circ} \mathrm{C}$ or more for typical current pulses [7, 8]. The fraction of atoms capable of being captured by a typical MOT is quite small $\left(\approx 10^{-5}\right)$ due to the large temperatures reached, but the efficacy of the dispensers for direct

*Electronic address: klmoore@socrates.berkeley.edu loading is salvaged both by the large atom flux and their ability to be placed close to the MOT. Alkali metal dispensers are already used as sources for vapor cell MOTs (Refs 9, 10, 11] for example), but in these cases the 800 ${ }^{\circ} \mathrm{C}$ atoms are cooled by the walls of the vapor cell so the loading rate into the MOT is increased.

Many ultracold atomic physics experiments demand base pressures of less than $10^{-10}$ torr, particularly in the case of magnetic trapping of atoms for long periods of time. Getters have been used directly in an ultra-high vacuum (UHV) ultracold atom experiment 12 and the atomic flux from a dispenser has been collimated to make an atom beam 13], but to the best of the authors' knowledge no published work describes a system which attempts to control the output flux of the getter entirely in the UHV chamber.

The desire for a fast, simple, and efficient source of rubidium atoms with a minimal impact on UHV conditions led us to the development of a cold shroud for the rubidium dispenser and MOT system. The shroud acts as a pump for rubidium atoms released by the dispenser that are either (a) not directed towards the MOT, (b) of the wrong isotope, or (c) moving too quickly to be captured by the MOT. The dispenser-shroud system thus acts as a fast, compact, collimated atomic beam source with a minimized impact on UHV conditions.

This article discusses the performance of the gettershroud system as well as the efficiency of loading atoms into a MOT from the direct flux of a dispenser. Importantly, our measurements characterizing the loading rate and equilibrium populations of a MOT indicate that direct loading of atoms from a getter is strikingly ineffective. In contrast, our measurements indicated that a secondary, lower temperature atomic source was also formed, contingent on operation of the getter, which was much more effective at loading a MOT at UHV conditions. Future getter-loaded, UHV experiments can be designed to make use of this tempered source in a more controlled manner. 


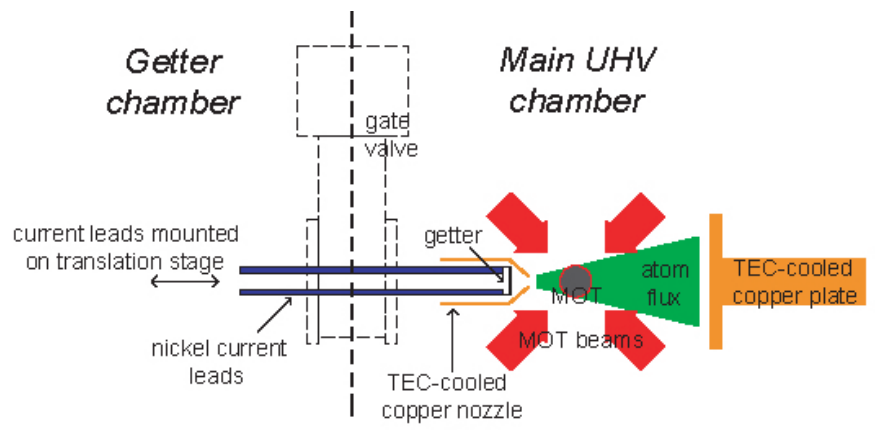

FIG. 1: Essential elements of the getter and cold shroud system. The getter is brought within 1.2 inches of the MOT center, and with a driving current greater than $2.7 \mathrm{~A}$ it releases a hot rubidium vapor. The cold copper nozzle that surrounds the getter absorbs nearly all of the emitted rubidium atoms that are not directed through the open aperture towards the MOT. The MOT is loaded from the resultant atomic beam exiting the nozzle. The vast majority of emitted atoms are moving too quickly to be captured by the MOT, but are absorbed by the cold copper plate on the right. The shroud is comprised of the copper nozzle, the copper plate, and surrounding mechanical structure.

\section{EXPERIMENT}

Our experiments are carried out in a stainless steel UHV chamber pumped to below $10^{-10}$ torr. This chamber was divided between a main chamber and a secondary "getter chamber," designed so as to allow defective or depleted getters to be replaced without exposure of the main UHV chamber to atmosphere. The getter is spotwelded to nickel rods and mounted on a hollow linear feedthrough [14] which provides six inches of travel. The current feedthrough also has a hollow interior (0.4" ID tube) and a 1.33 inch mini-flange port which accepts an electrical feedthrough to control the current through the getter. A single-rod current feedthrough is sufficient, as the return current path can be grounded to the chamber on the interior bellows of the linear motion feedthrough.

Figure 1 shows a schematic of the experiment. The mounted getter on a retracted translation stage rests just behind a gate valve to the main chamber. The vacuum region that surrounds the dispenser and translation stage is evacuated by a turbomolecular and an ion pump. Once this region is opened, it takes less than 30 minutes to spot weld a new getter and reseal the vacuum system. The "getter chamber" is then evacuated and undergoes a modest bakeout ( $\approx 2$ days), during which the dispenser is degassed as discussed by the authors of Ref. 7]. We typically follow their procedure, although the alternate method advocated by Ref. [8] also provides a usable rubidium source.

When the gate valve is opened, pressures in the $10^{-10}$ torr range are established in both the getter chamber and the main chamber. With the entry path though the gate valve clear, the dispenser can be translated to within 1.25

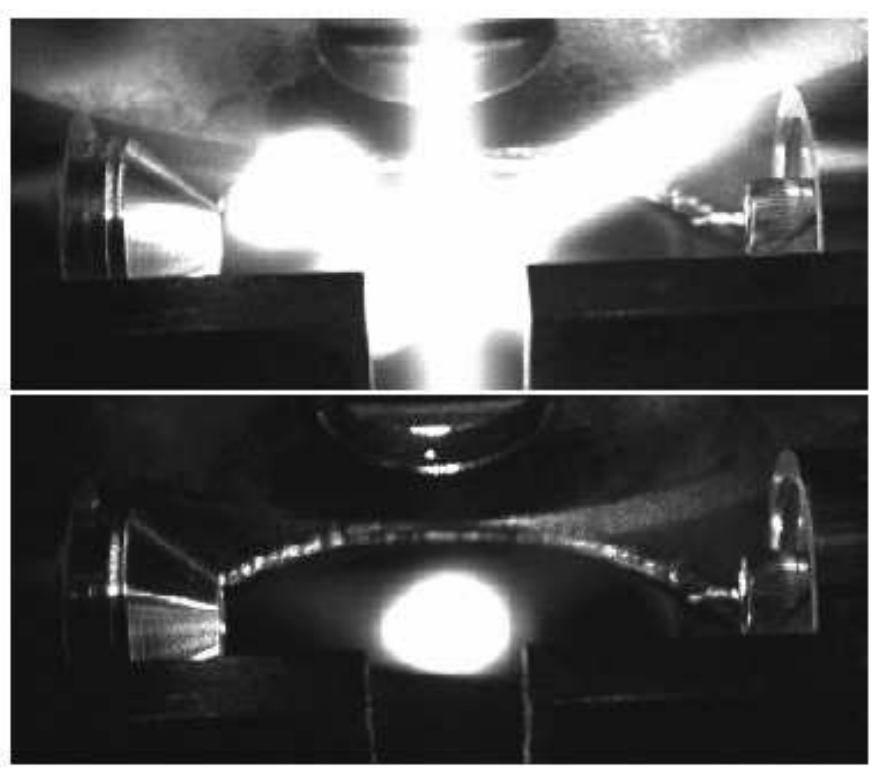

FIG. 2: Extinction of a background vapor and production of a single-pass atomic beam by low temperature shroud. The top image shows the fluorescence of the background rubidium atoms for a getter pulse of $10 \mathrm{~A}$ for 15 seconds at a shroud temperature of $21.5^{\circ} \mathrm{C}$. The bottom image is the fluorescence for an identical getter pulse and incident MOT beams, but instead the shroud is held at $-30^{\circ} \mathrm{C}$. Note the sharp edges of the atom beam, as well as the disappearance of background fluorescence. In both images, the atom beam flows from left to right, consistent with the orientation of Fig. 1.

inches of the MOT center.

To ensure that rubidium atoms not captured by the MOT are pumped away, all line of sight from the dispenser to the room temperature UHV chamber is blocked by a nickel-plated cold copper shroud. As the shroud is cooled, the sticking probability for a rubidium atom (or any alkali metal) impacting the surface approaches unity. In theory this would protect all sensitive surfaces from the direct flux of atoms as well as preventing a room temperature background vapor of rubidium from permeating the chamber during an experiment. Figure 2 highlights the suppression of the background rubidium vapor with the cooling of the cold shroud; operating the getter while the shroud is at room temperature produces visible fluorescence throughout the chamber due to a thermal rubidium vapor, while similar operation with a cold $\left(-30{ }^{\circ} \mathrm{C}\right)$ shroud yields fluorescence only from a beam with line-ofsight access to the getter. The pressure spike associated with the dispenser heating, observable at $10^{-10}$ torr with an ion gauge, is also reduced by nearly a factor of two with the cooling of the shroud.

Atoms are collected in a MOT centered within the getter-emitted beam. The MOT is formed from $13 \mathrm{~mW}$ of laser power to each of six 0.75 inch diameter beams. The quadrupole coils are placed outside the vacuum system, three inches from the center of the MOT, and are typically operated with axial field gradients of $20 \mathrm{G} / \mathrm{cm}$. 


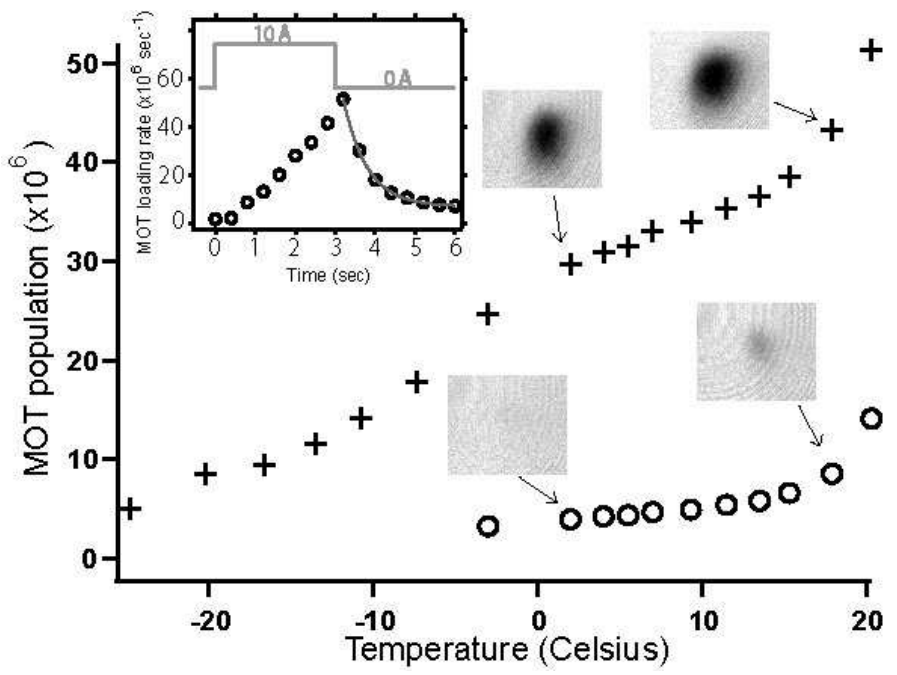

FIG. 3: MOT population as a function of shroud temperature. Crosses $(+)$ denote a MOT loaded by a $10 \mathrm{~A}, 6 \mathrm{sec}$ pulse, circles (o) a MOT loaded from background atoms for 6 sec with no getter pulse. Inset shows MOT loading rate as a function of time overlayed upon the current profile of a $3 \mathrm{sec}$, 10 A getter pulse (the shroud temperature was $19^{\circ} \mathrm{C}$ ). The rapid quenching of the MOT loading rate upon cessation of the getter current has a decay time of $0.6 \mathrm{sec}$.

The MOT population was limited by the relatively small amount of light power per beam. The trends reported in this paper should scale directly with improvements in MOT loading.

\section{RESULTS}

Our pulsed atomic source should be evaluated under two criteria: first, that operating the source yields an atomic flux which is efficiently loaded into sizeable MOTs, and, second, that the source can be quickly switched off to yield UHV conditions for subsequent experimentation (e.g. magnetic trapping and evaporation) of the laser-cooled atoms. We assessed the performance of our getter-shroud system under both criteria by measuring the loading rate and equilibrium population in MOTs formed either during or after the atomic source was pulsed: large MOTs formed during the getter pulse indicate efficient loading, while small MOTs formed seconds after the getter pulse indicate the desired suppression of the atomic flux when the source is turned off. Measurements of MOT populations under both conditions (getter on/getter off) are shown in Figure 3 as a function of the temperature of the shroud.

Several conclusions can be drawn from this data. First, at all temperatures of the shroud, the MOT populations formed through operating the getter are much higher than those collected from the background atomic flux, indicating that the getter-shroud system is indeed operating as a pulsed atomic beam source, as desired. Longer getter pulses make use of this high loading rate to reach equilibrium MOT numbers up to $3 \times 10^{8}$. Second, confirming the visual findings of Fig. 2, the cold shroud extinguishes the background rubidium vapor if it is operated at sufficiently low temperatures; below $0{ }^{\circ} \mathrm{C}$, the MOTs formed from the background vapor were only barely detected by our optical absorption measurements. Further, the inset of Fig. 3 shows the rapid termination of the MOT loading rate upon extinguishing the getter current, satisfying the second stated criterion for the system.

While the getter-shroud system satisfies the stated criteria as an efficient, rapidly-switched atom source, this data exhibits some surprising features. The reason that the getter-loaded MOT depends so strongly on the shroud temperature, including an almost complete elimination of trapped atoms for the coldest temperatures, is not immediately obvious. The dispenser assembly has no mechanical contact with the shroud, meaning that the getter itself arrives at the same temperatures regardless of the temperature of the shroud. The small MOT populations for low shroud temperatures suggest that the direct atom flux from the getter is actually a rather poor source for a MOT. Our original intent was to utilize this shroud at or below $-20{ }^{\circ} \mathrm{C}$, but the reduced MOT population at lower temperatures forces a choice between rubidium background elimination and larger MOT populations. The optimum shroud temperature will likely vary for different experimental requirements.

In order to diagnose the thermal character of atoms emitted by the dispenser, a laser $45^{\circ}$ to the atomic beam was scanned in frequency while fluorescence at the center of the MOT region was detected on a photodiode. This yields information about the velocity distribution of the emitted atoms, though the resultant spectrum is expected to be a convolution of many competing factors due to the large probe beam size $(\approx 0.75$ inch diameter $)$, divergence of emitted atoms, optical pumping rates, and background fluorescence.

The fluorescence data (Figure 4) show the effect of the cold shroud on the velocity distribution of the emitted atoms from a strong getter pulse $(10 \mathrm{~A}, 30 \mathrm{sec})$. For a shroud at room temperature the overall rubidium density in the MOT region is approximately twice that of a -11 ${ }^{\circ} \mathrm{C}$ shroud. At $21{ }^{\circ} \mathrm{C}$ the background rubidium vapor and the rubidium flux from the nozzle can be clearly distinguished, and the Doppler-shifted atoms show peak fluorescence at $550 \mathrm{MHz}$ from the background vapor. At $-11{ }^{\circ} \mathrm{C}$, the background atoms are barely resolvable, and the Doppler-shifted atoms are now peaked at $630 \mathrm{MHz}$. Also plotted in Fig. 4 is the fluorescence curve for a room temperature $\left(2{ }^{\circ} \mathrm{C}\right)$ shroud and no getter flux. In this case, the peak fluorescence in the MOT region is shifted by $300 \mathrm{MHz}$ from the line center of the Dopplerbroadened background rubidium spectrum. We believe this is caused by the flux of desorbing atoms from the shroud nozzle surface which acts as a directed, roomtemperature background flux into the MOT region; we 


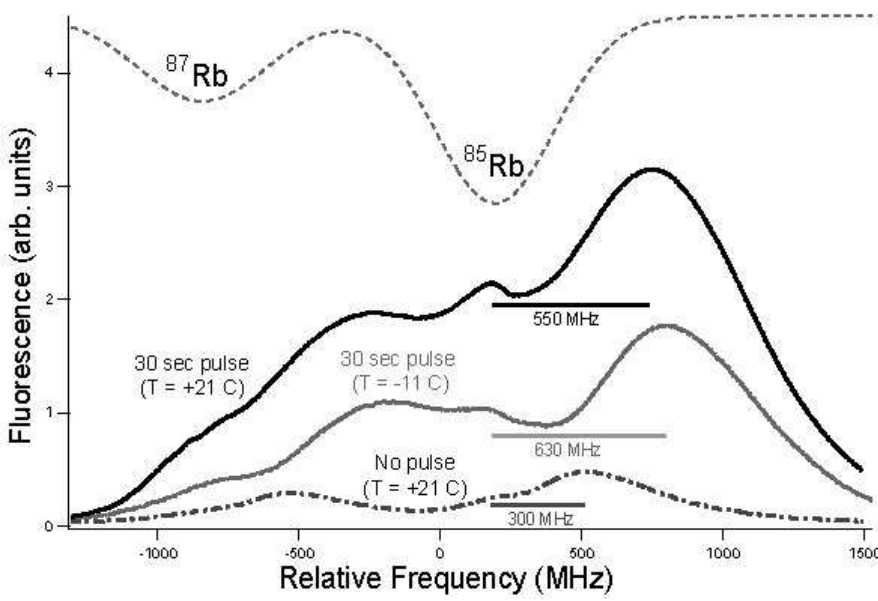

FIG. 4: Doppler-sensitive fluorescence spectra of atom flux in the MOT region. The probe laser beam was incident upon the atom flux at $45^{\circ}$ to the center line of the the shroud nozzle. For reference, the dotted line shows the room temperature rubidium spectrum, inferred from an absorption signal generated in a vapor cell. Plotted are fluorescence curves when the shroud is at room temperature and at $-12{ }^{\circ} \mathrm{C}$, given a 30 second, $10 \mathrm{~A}$ current pulse to the getter. Also plotted is the background fluorescence in the MOT region with no getter pulse, clearly showing the sizeable room temperature flux emitted from the room temperature shroud surface after a large layer of atoms have desorbed on the inner surface of the nozzle.

were only able to discern this fluorescence spectrum after an atypically large layer of rubidium had been deposited on the inner surface of the shroud nozzle.

These data explicitly demonstrates that the velocity distribution of the atomic flux through the nozzle and into the MOT region is significantly affected by the thermal state of the shroud. The strong dependence of the getter-loaded MOT population on shroud temperature in Fig. 3 is better understood in this context, especially given the fact that a room temperature beam loads $\approx 10$ times more atoms into a MOT than a $1000 \mathrm{~K}$ oven of the same number flux. The most likely explanation for the modification of the atom flux is that a large fraction of the atomic flux from the getter is impingent upon the cold nozzle used to collimate the remaining flux. If the surface has a substantial probability of reflecting and thermalizing these atoms, then a flux seemingly colder than the getter-only flux seen at $-11{ }^{\circ} \mathrm{C}$ will be emitted because the emitted atoms will be a mixture of hot getter-emitted atoms and cooler reflected atoms. The thermalization of getter-emitted atoms with surrounding walls has been previously observed [13]. The sum of these two fluxes yields the reduced Doppler shift seen in the room temperature data in Fig. 4. We found that after a few weeks of normal operation of the getter the desorbing flux would then persist for several days.

\section{DISCUSSION}

While the dispenser-shroud system has proven itself to be potentially useful for ultracold atomic experiments, there are some drawbacks. We tried many different avenues for continuous operation in an attempt to maximize MOT population and minimize the experiment repetition time. If the getter is pulsed too frequently $(<20$ seconds separation between $3 \mathrm{sec}, 10 \mathrm{~A}$ pulses) the pressure in the chamber rises to a steady state above the minimum base pressure. While we believe this to be an improvement over bare operation of a dispenser in UHV, we had hoped to reduce this repetition time further. However, even with the coldest shrouds that the TEC system could effect we were unable to execute an experimental cycle of less than 20 seconds.

Second, rubidium adsorbed on the shroud will be released into the UHV if the shroud is allowed to warm up. If one is to prevent this substantial gas load from interfering with in-vacuum equipment (such as ion pumps or high-finesse mirrors), the cooling of the shroud must be made fail safe. Furthermore, one would not be able to bake the shroud in UHV. Thus contamination of the UHV chamber would be correctable only by selective baking or by thoroughly cleaning the chamber. This disadvantage belies the purpose of the getter as an easily-exchanged UHV atom source.

Drawbacks aside, several improvements to our design could be made which would make the system a useful tool in many instances. First, the aperture on the nozzle could be widened to allow a larger flux into the MOT. The aperture is currently 0.25 " in diameter and appears to "choke" the MOT at lower temperatures because the beam is only shining into a fraction of the MOT crosssection. A larger aperture would increase the flux as the square of the aperture diameter, allowing the maximum getter-loaded population number to be reached in less time with a reduced impact on the pressure.

Another improvement would be the addition of a "shadow" for the getter. This would likely take the form of a metal piece which would obscure the getter slit from direct line of sight to the center of the MOT. This should drastically reduce the losses due to MOT atoms colliding with fast $\mathrm{Rb}$ atoms, allowing for a larger final MOT population.

Finally, given our understanding of the fluorescence spectrum in Fig. 4, one could construct a system which would utilize the secondary room temperature beam generated by the rubidium-coated surfaces of the shroud. A miniature "oven," operated in the main UHV chamber, could surround a dispenser that purposefully directs its atomic flux towards the inner walls of the oven. Atoms emitted from the getter would be tempered by the inner surface of the oven, and these thermalized atoms would then be allowed to escape through a collimated aperture to efficiently load a closely situated MOT. Cold baffles would then be placed behind the MOT to pump away the atoms which are not captured by the MOT. During ex- 
perimental operation the oven could be held at or slightly above room temperature to increase atom yield and prevent a surface layer of atoms from forming. When the experimental system is not in operation the miniature oven could be heated further to release any remaining adsorbed atoms onto the cold baffles.

\section{Acknowledgments}

We thank Stefan Schmid, Mike Grobis, and Dave Murai for their technical assistance and advice. The authors effort was sponsored by the Defense Advanced Research Projects Agency (DARPA) and Air Force Laboratory, Air Force Materiel Command, USAF, under Contract No. F30602-01-2-0524, the National Science Foundation under Grant No. 0130414, the Sloan Foundation, the David and Lucile Packard Foundation, and the University of California. KLM acknowledges support from the National Science Foundation. SG acknowledges support from the Miller Institute for Basic Research in Science.
[1] N. F. Ramsey, Molecular Beams. Oxford University Press, Reprint Ed. (1997).

[2] W. D. Phillips and H. Metcalf, Phys. Rev. Lett. 48, 596 (1982).

[3] C. J. Myatt et al., Optics Letters 21, 290 (1996).

[4] B. P. Anderson and M. A. Kasevich, Phys. Rev. A 64, 023404 (2001).

[5] S. N. Atutov et al., Phys. Rev. A 67, 053401 (2003).

[6] Manufactured by SAES Getters USA Inc.

[7] J. Fortagh, A. Grossman, T. W. Hansch, and C. Zimmermann, J. App. Phys 84, 6499 (1998).

[8] U. D. Rapol, A. Wasan, and V. Natarajan, Phys. Rev. A 64, 023402 (2001).
[9] H. J. Lewandowski, Ph.D. thesis, University of Colorado (2002)

[10] M. R. Matthews et al., Phys. Rev. Lett. 81, 243 (1998).

[11] C. Wieman, G. Flowers, and S. Gilbert, Am. J. Phys. 63, 317 (1995).

[12] H. Ott et al., Phys. Rev. Lett. 87, 230401 (2001).

[13] T. M. Roach and D. Henclewood, to be published in Jour. of Vac. Sci. and Tech. A.

[14] Manufactured by Thermionics Vacuum Products, part number FLMH-275-50-6. Inclusion of this item is not an endorsement; we expect that devices from other manufacturers would behave in a similar fashion. 